\title{
Reheating the Universe in Braneworld Cosmological Models with bulk-brane energy transfer
}

\author{
T. Harko* W. F. Choi, K. C. Wong, and K. S. Cheng \\ Department of Physics and Center for Theoretical and Computational Physics, \\ University of Hong Kong, Pok Fu Lam Road, Hong Kong, P. R. China
}

(Dated: September 16, 2021)

\begin{abstract}
The emergence of the cosmological composition (the reheating era) after the inflationary period is analyzed in the framework of the braneworld models, in which our Universe is a three-brane embedded in a five-dimensional bulk, by assuming the possibility of the brane-bulk energy exchange. The inflaton field is assumed to decay into normal matter only, while the dark matter is injected into the brane from the bulk. To describe the reheating process we adopt a phenomenological approach, by describing the decay of the inflaton field by a friction term proportional to the energy density of the field. After the radiation dominated epoch the model reduces to the standard four dimensional cosmological model. The modified field equations are analyzed analytically and numerically in both the extra-dimensions dominate reheating phase (when the quadratic terms in energy density dominate the dynamics), and in the general case. The evolution profiles of the matter, of the scalar field and of the scale factor of the universe are obtained for different values of the parameters of the model, and of the equations of state of the normal and dark matter, respectively. The equation describing the time evolution of the ratio of the energy density of the dark and of the normal matter is also obtained. The ratio depends on the rate of the energy flow between the bulk and the brane. The observational constraint of an approximately constant ratio of the dark and of the baryonic matter requires that the dark matter must be non-relativistic (cold). The model predicts a reheating temperature of the order of $3 \times 10^{6} \mathrm{GeV}$, a brane tension of the order of $10^{25} \mathrm{GeV}^{4}$, and the obtained composition of the universe is consistent with the observational data.

PACS numbers: 04.50.-h, 04.20.Jb, 04.20.Cv, 95.35.+d
\end{abstract}

*Electronic address: harko@hkucc.hku.hk 


\section{INTRODUCTION}

Early studies on superstring theory and M-theory have suggested that our four dimensional world is embedded into a higher dimensional spacetime. Particularly, the 10 dimensional $E_{8} \otimes E_{8}$ heterotic superstring theory is a low-energy limit of the 11 dimensional supergravity, under the compactification scheme $M^{10} \times S_{1} / Z_{2}$ [1, 2]. Thus, the 10 dimensional spacetime is compactified as $M^{4} \times C Y^{6} \times S_{1} / Z_{2}$, implying that our universe (a brane) is embedded into a higher dimensional bulk. In this paradigm, the standard model particles are open strings, confined on the braneworld, whilst the gravitons and the closed strings can freely propagate into the bulk [3].

Among the collection of the braneworld scenarios, the Randall-Sundrum Type II model has the virtue of providing a new type of compactification of gravity [4, 5]. Standard 4D gravity can be recovered in the low-energy limit of the model, with a 3-brane of positive tension embedded in 5D anti-de Sitter bulk. The covariant formulation of the brane world models has been formulated in [6], leading to the modification of the standard Friedmann equations on the brane. It turns out that the dynamics of the early universe is altered by the quadratic terms in the energy density and by the contribution of the components of the bulk Weyl tensor, which both give a contribution in the energy momentum tensor. This implies a modification of the basic equations describing the cosmological and astrophysical dynamics, which has been extensively considered recently [7].

The recent observations of the CMB anisotropy by WMAP [8] have provided convincing evidence for the inflationary paradigm [9], according to which in its very early stages the universe experienced an accelerated (de Sitter) expansionary phase (for recent reviews on inflation see [10]).

At the end of inflation, the universe is in a cold and low-entropy phase, which is utterly different from the present hot high-entropy universe. Therefore the universe should be reheated, or defrosted, to a high enough temperature, in order to recover the standard Hot Big Bang [11]. The reheating process may be envisioned as follows: the energy density in zero-momentum mode of the scalar field decays into normal particles with decay rate $\Gamma$. The decay products then scattered and thermalize to form a plasma [10].

Apart from the behavior of the inflaton field, the evolutions of dark energy and dark matter in reheating stage were also considered. In [12], dark energy and dark matter were 
originated from a scalar field in different stages of the inflation, according to a special form of potential. Meanwhile, the conditions for unifying the description of inflation, dark matter and dark energy were considered in [13]. A specific model was later proposed in [14], by using a modified quadratic scalar potential. The candidates of dark matter in [13] and [14] were oscillations of a scalar field. However, it may be possible that dark matter existed on its own without originating from the scalar field. This may pose less stringent constraint on the scalar field, so that dark matter can be included in inflation paradigm in a easier way. On the other hand, it was proposed that the decay products of scalar field acquired thermal mass [15].

The reheating in the braneworld models has also been considered recently. In the context of the braneworld inflation driven by a bulk scalar field, the energy dissipation from the bulk scalar field into the matter on the brane was studied in [16]. The obtained results supports the idea that the brane inflation model, caused by a bulk scalar field, may be a viable alternative scenario of the early universe. The inflation and reheating in a brane world model derived from Type IIA string theory was studied in [17]. In this model the inflaton can decay into scalar and spinor particles, thus reheating the universe. A model in which high energy brane corrections allow a single scalar field to describe inflation at early epochs and quintessence at late times was discussed in [18]. The reheating mechanism in the model originates from Born-Infeld matter, whose energy density mimics cosmological constant at very early times and manifests itself as radiation subsequently. The particle production at the collision of two domain walls in a 5-dimensional Minkowski spacetime was studied in [19]. This may provide the reheating mechanism of an ekpyrotic (or cyclic) brane universe, in which two BPS branes collide and evolve into a hot big bang universe. The reheating temperature $T_{R H}$ in models in which the universe exits reheating at temperatures in the $\mathrm{MeV}$ regime was studied in [20], and a minimum bound on $T_{R H}$ was obtained. The derived lower bound on the reheating temperature also leads to very stringent bounds on the compactification scale in models with $n$ large extra dimensions. The dark matter problem in the Randall-Sundrum type II brane world scenario was discussed in [21], by assuming that the lightest supersymmetric particle is the axino. The axinos can play the role of cold dark matter in the universe, due to the higher reheating temperatures in the brane world model, as compared to the conventional four-dimensional cosmology. The impact of the non-conventional brane cosmology on the relic abundance of non-relativistic stable particles 
in high and low reheating scenarios was investigated in [22]. In the case of high reheating temperatures, the brane cosmology may enhance the dark matter relic density by many order of magnitudes, and a stringent lower bound on the five dimensional scale may be obtained. In the non-equilibrium case, the resulting relic density is very small. The curvaton dynamics in brane-world cosmologies was studied in [23]. By assuming that the inflaton field survives without decay after the end of inflation, the curvaton reheating mechanism was applied to the Randall-Sundrum model and to its curvature corrections.

Recently, motivated by particle physics results, the possibility of an energy exchange between the bulk and the brane was also suggested. The observational constraints on the cosmological models in the brane-world scenario in which the bulk is not empty, and exchange of mass-energy between the bulk and the bane is allowed, were explored in [24]. The evolution of matter fields on the brane is modified due to new terms in the energy momentum tensor describing this exchange. This model accounts for the observed suppression of the CMB power spectrum at low multipoles, and the observed recent cosmic acceleration is attributable to the flow of matter from the bulk to the brane. The cosmological evolution of a brane with a general bulk matter content was considered in [25], by assuming that the bulk pressure and the energy exchange densities are comparable to the brane energy density. By adopting a phenomenological fluid ansatz and generalizations of it, a set of exact solutions of the Friedmann equation that exhibit accelerated expansion were derived. The braneworld models with bulk-brane energy exchange allow for crossing of the $w=-1$ phantom divide line without introducing phantom energy with quantum instabilities [26]. The class of the braneworld models with bulk-brane energy exchange have the ability to provide crossing of the phantom divide, and the observational cosmological data hint towards natural values for the model parameters.

It is the purpose of the present paper to construct a model of braneworld reheating by adopting as a key feature the energy exchange between the bulk and the brane during the reheating stage. The era of focus will be from the end of inflation until the present time, in contrast with [24] and [25, 26]. In order to explain the present day composition of our universe, we adopt, as an essential characteristic of our model, the hypothesis that the existence of the dark matter is the result of the real matter exchange between the bulk and the brane. However, the other matter fields bare no such freedom, according to the confinement conditions introduced in [3] . 
The main physical features of our model can be presented as follows: the dark matter from the bulk and flows to the brane after the inflationary phase, while the scalar fields and the normal matter are always confined on the brane. Therefore the bulk leaves an imprint on the brane by injecting dark matter. The effect of dark matter flowing into the brane is studied in the context of the cosmological composition, with the reheating temperature as an important parameter. The inflaton field decays into the normal matter field [11, 27], attaining thermal equilibrium and acquiring plasma masses through the mechanism proposed in [15]. Therefore, as reheating is concerned, the presence of the scalar field is essential, and it decays into visible matter only. Furthermore, the specific forms of the inflaton potential are not essential in our approach, since during reheating, the kinetic term $\dot{\phi}^{2}$ plays a crucial role. The main focus of the present paper is to study the dynamics of the universe by taking into account the extra-dimensional effects on the reheating process. The ratio $u$ of the energy densities of the dark matter and of the normal matter is an important parameter of our model, and its time evolution is studied by using both analytical and numerical methods. As a test of the model, several important observational parameters like the maximum density of the matter in the universe and the reheating temperature $T_{r e h}$ are also estimated. Hence, we adopt as our main observational constraints the constancy of the ratio $u$ after the photon decoupling, the absence of the inflaton in the present day universe, and the bound of $T_{r e h}$ from gravitino product, [28, 29, 30].

The present paper is organized as follows. The basic equations of our model are formulated in Section II. A dimensionless form of the field equations is presented in Section III, where the physically acceptable range of the values of the physical parameters is also discussed. The extra-dimensional effects dominated phase of the reheating, during which the quadratic terms in the energy density dominate the dynamics and evolution of the universe, is discussed in Section IV. The reheating process is considered in its full generality in Section $\mathrm{V}$, and the role of the linear terms in the energy density is investigated. Finally, in Sec. VI we summarize and conclude our results. 


\section{GEOMETRY AND FIELD EQUATIONS IN THE BULK-BRANE ENERGY TRANSFER MODEL}

We start by considering a five dimensional $(5 D)$ spacetime (the bulk), with a single fourdimensional (4D) brane, on which usual (baryonic) matter and physical fields are confined. The $4 D$ brane world $\left({ }^{(4)} M, g_{\mu \nu}\right)$ is located at a hypersurface $\left(B\left(X^{A}\right)=0\right)$ in the $5 D$ bulk spacetime $\left({ }^{(5)} M, g_{A B}\right)$, of which coordinates are described by $X^{A}, A=0,1, \ldots, 4$. The induced $4 D$ coordinates on the brane are $x^{\mu}, \mu=0,1,2,3$.

The action of the system is given by [6]

$$
S=S_{\text {bulk }}+S_{\text {brane }}
$$

where

$$
S_{\text {bulk }}=\int_{(5) M} \sqrt{-{ }^{(5)} g}\left[{\frac{1}{2 k_{5}^{2}}}^{(5)} R+{ }^{(5)} L_{m}+\Lambda_{5}\right] d^{5} X,
$$

and

$$
S_{\text {brane }}=\int_{{ }_{(4)} M} \sqrt{-^{(5)} g}\left[\frac{1}{k_{5}^{2}} K^{ \pm}+L_{\text {brane }}\left(g_{\alpha \beta}, \phi\right)+\lambda\right] d^{4} x,
$$

where $k_{5}^{2}=8 \pi G_{5}$ is the $5 D$ gravitational constant, ${ }^{(5)} R$ and ${ }^{(5)} L_{m}$ are the $5 D$ scalar curvature and the matter Lagrangian in the bulk, $L_{\text {brane }}\left(g_{\alpha \beta}, \phi\right)$ is the $4 \mathrm{~d}$ Lagrangian, which is given by a generic functional of the brane metric $g_{\alpha \beta}$ and of the matter fields $\phi, K^{ \pm}$is the trace of the extrinsic curvature on either side of the brane, and $\Lambda_{5}$ and $\lambda$ (the constant brane tension) are the negative vacuum energy densities in the bulk and on the brane, respectively. The brane tension is the characteristic energy scale of the brane, below which the quadratic terms in the field equations, derived from the action Eq. (1), can be neglected. Thus, in the limit $\lambda \rightarrow \infty$ the field equations reduce to the usual form of the standard general relativity.

The Einstein field equations in the bulk are given by [ 6 ]

$$
{ }^{(5)} G_{I J}=k_{5}^{2(5)} T_{I J}, \quad{ }^{(5)} T_{I J}=-\Lambda_{5}{ }^{(5)} g_{I J}+\delta(B)\left[-\lambda^{(5)} g_{I J}+T_{I J}\right],
$$

where

$$
{ }^{(5)} T_{I J} \equiv-2 \frac{\delta^{(5)} L_{m}}{\delta^{(5)} g^{I J}}+{ }^{(5)} g_{I J}{ }^{(5)} L_{m},
$$

is the energy-momentum tensor of bulk matter fields, while $T_{\mu \nu}$ is the energy-momentum tensor localized on the brane and which is defined by

$$
T_{\mu \nu} \equiv-2 \frac{\delta L_{\text {brane }}}{\delta g^{\mu \nu}}+g_{\mu \nu} L_{\text {brane }} .
$$


The delta function $\delta(B)$ denotes the localization of brane contribution. In the $5 D$ spacetime a brane is a fixed point of the $Z_{2}$ symmetry. In the following we assume

$$
{ }^{(5)} L_{m} \neq 0 \text {. }
$$

The induced $4 D$ metric is $g_{I J}={ }^{(5)} g_{I J}-n_{I} n_{J}$, where $n_{I}$ is the space-like unit vector field normal to the brane hypersurface ${ }^{(4)} M$. The basic equations on the brane are obtained by projections onto the brane world, and are given by [31]

$$
G_{\mu \nu}=-3 \lambda g_{\mu \nu}+\frac{\lambda^{(5)}}{24 k_{5}^{4}} T_{\mu \nu}+\frac{1}{4 k_{5}^{4}} S_{\mu \nu}-\varepsilon_{\mu \nu}+\frac{1}{3 k_{5}^{4}} F_{\mu \nu},
$$

where

$$
\begin{gathered}
S_{\mu \nu}=\frac{1}{2} T T_{\mu \nu}-\frac{1}{4} T_{\mu \alpha} T_{\nu}^{\alpha}+\frac{3 T_{\alpha \beta} T^{\alpha \beta}-T^{2}}{24} g_{\mu \nu}, \\
\varepsilon_{\mu \nu}=C_{A B C D} n^{C} n^{D} g_{\mu}^{A} g_{\nu}^{B},
\end{gathered}
$$

and

$$
F_{\mu \nu}={ }^{(5)} T_{A B} g_{\mu}^{A} g_{\nu}^{B}+\left({ }^{(5)} T_{A B} n^{A} n^{B}-\frac{1}{4}^{(5)} T\right) g_{\mu \nu},
$$

respectively.

Apart from the terms quadratic in the brane energy-momentum tensor, in the field equations on the brane there are two supplementary terms, corresponding to the projection of the $5 D$ Weyl tensor $\varepsilon_{\mu \nu}$ and of the projected tensor $F_{\mu \nu}$, which contains the bulk matter contribution. Both terms induce bulk effects on the brane.

For cosmological applications we adopt a metric of the form

$$
d s^{2}=-n^{2}(t, \xi) d t^{2}+a^{2}(t, \xi) \gamma_{\mu \nu} d x^{\mu} d x^{\nu}+d \xi^{2},
$$

where $\gamma_{\mu \nu}$ is the maximally symmetric 3-space, and we denoted the fifth coordinate by $x^{5}=\xi . a$ and $n$ are the scale and shift factors, respectively. The Hubble parameter $H$ on the brane, describing the cosmological dynamics of the universe, is defined as $H=\dot{a} / a$. For further discussions on the gravitational field equations in the brane world models and their cosmological applications see [32, 33, 34, 35, 36, 37].

On the brane the matter, consisting of normal matter, dark matter, a scalar field etc., is in a perfect fluid form, and is described in terms of a total energy density $\rho_{\text {total }}=\sum_{i=1}^{n} \rho_{i}$, where $\rho_{i}, i=1, \ldots, n$ are the energy densities of the individual components, and a total 
thermodynamic pressure $p_{\text {total }}=\sum_{i=1}^{n} p_{i}$, where $p_{i}, i=1, \ldots, n$ are the pressures of the matter fluid components. The corresponding energy-momentum tensor on the brane is

$$
T_{\nu}^{\mu}=\delta(\xi) \operatorname{diag}\left(-\rho_{\text {total }}, p_{\text {total }}, p_{\text {total }}, p_{\text {total }}\right) .
$$

The delta function $\delta(\xi)$ implies the confinement of the matter fields on the brane at $\xi=0$.

We assume that the matter component in the bulk is in the form of dark matter, with energy density $\rho_{D M}$ and pressure $p_{D M}$, respectively, with energy-momentum tensor

$$
T_{B}^{A}=\left(\rho_{D M}+p_{D M}\right) U^{A} U_{B}+p_{D M} \delta_{B}^{A}
$$

Then the energy momentum tensor $T_{B}^{A}$ of dark matter in the bulk has the non-zero components :

$$
T_{5}^{0} \sim\left(\rho_{D M}+p_{D M}\right) U_{5}, T_{5}^{5}=\left(\rho_{D M}+p_{D M}\right) U^{5} U_{5}+p_{D M},
$$

where the five-velocity $U_{5}$ represents the matter flow from the bulk to the brane [24]. Hence in our model the dark matter can exist simultaneously in the bulk and on the brane.

The term $2 T_{5}^{0}$ is the discontinuity of the $(0,5)$ component of energy momentum tensor at $\xi=0$. In the static bulk with respect to the expanding brane, $U_{5}$ is given by $U_{5} \propto-l H$, where $l=\sqrt{-6 M_{5}^{3} / \Lambda_{5}}$ is the bulk curvature radius, and $M_{5}$ and $\Lambda_{5}$ are the Planck mass and the cosmological constant in the 5 dimensional bulk, respectively [38, 39]. We assume that the dark matter obeys an equation of state of the form

$$
p_{D M}=(w-1) \rho_{D M},
$$

where $1 \leq w \leq 2$ is a constant.

To obtain $T_{5}^{0}$ we follow the approach developed in [24]. Since $\rho_{D M}=\rho_{c r}\left(a_{0} / a\right)^{3 w}$, where $\rho_{c r}$ is the present day critical density and $a_{0}$ is the value of the scale factor at the moment when the energy transfer from the bulk to the brane begins, we obtain

$$
T_{5}^{0}=-\frac{\alpha_{b b}}{2} \rho_{c r}\left(\frac{a_{0}}{a}\right)^{3 w} H .
$$

The dimensionless parameter $\alpha_{b b}$ describes the flow of dark matter from bulk to brane. Finally, the evolution equation of the dark matter on the brane is [24]

$$
\dot{\rho}_{D M}+3 H\left(\rho_{D M}+p_{D M}\right)=-2 T_{5}^{0}
$$


In the widely accepted inflationary scenario it is assumed that during an initial period the universe is dominated by a large, approximately constant potential term $V(\phi)$ of a scalar field $\phi$, known as the inflaton field [9, 10]. The energy-momentum tensor of the scalar field can be written in the perfect fluid form, with energy density $\rho_{\phi}$ and pressure $p_{\phi}$ given by

$$
\rho_{\phi}=\frac{\dot{\phi}^{2}}{2}+V(\phi),
$$

and

$$
p_{\phi}=\frac{\dot{\phi}^{2}}{2}-V(\phi)
$$

respectively. During a second period of evolution, the potential minimum is approached, $V(\phi)$ tends to zero, and the scalar field starts to fluctuate violently around the minimum value. The coupling to the field component becomes effective and the scalar field energy is converted into matter energy, thus giving rise to a large increase of the entropy [10, 11].

Inflationary scenarios have always to face the problem of the transition from a de Sitter stage, during which the evolution of the universe is dominated by the scalar field, to a subsequent radiation - or matter - dominated Friedmann-Robertson-Walker type cosmological model. One of the possible approaches to this problem is phenomenological [10, 11, 27], and consists in the introduction of a suitable loss term in the scalar field dynamics, which also appears as a source term for the energy density of the matter fluid. It is this source term which is responsible for the reheating process that follows the adiabatic supercooling process during the de Sitter phase. To describe mathematically the process is convenient to introduce a "friction" term $\Gamma$, describing the decay rate of the scalar field, and a corresponding term for the matter fluid.

Therefore, the basic equations describing the reheating phase after braneworld inflation, with matter exchange between the brane and the bulk, are given by

$$
\begin{gathered}
H^{2}=\left(\frac{\dot{a}}{a}\right)^{2}=\frac{8 \pi G \rho_{\text {total }}}{3}\left(1+\frac{\rho_{\text {total }}}{2 \lambda}\right)-\frac{k}{a^{2}}+\Lambda_{4}+\chi \\
\dot{\rho}_{m}+3 H\left(\rho_{m}+p_{m}\right)=\Gamma\left(\rho_{m}, \rho_{\phi}\right) \rho_{\phi}, \\
\dot{\rho}_{\phi}+3 H\left(\rho_{\phi}+p_{\phi}\right)=-\Gamma\left(\rho_{m}, \rho_{\phi}\right) \rho_{\phi} \\
\dot{\rho}_{D M}+3 H\left(\rho_{D M}+p_{D M}\right)=\frac{\alpha_{b b}}{2} \rho_{c r}\left(\frac{a_{0}}{a}\right)^{3 w} H \\
\dot{\chi}+4 \frac{\dot{a}}{a}\left(\chi+\frac{k_{5}^{2}}{6} T_{5}^{5}\right)=\frac{k_{5}^{4}}{9}\left(\rho_{\text {total }}+\lambda\right) T_{5}^{0}
\end{gathered}
$$




$$
\rho_{\text {total }}=\rho_{m}+\rho_{\phi}+\rho_{D M}
$$

where $\Lambda_{4}$ is the cosmological constant on the brane, $\chi$ is the so-called dark radiation term, and we have assumed the existence of a single matter component on the brane, with energy density $\rho_{m}$ and pressure $p_{m}$, respectively.

\section{DIMENSIONLESS FORM OF THE FIELD EQUATIONS}

The system of equations Eqs. (21)-(26) can be simplified by adopting some, physically justified, approximations. First of all, the $4 D$ cosmological constant $\Lambda_{4}$ can be neglected, as its derived value from the 3rd year WMAP data is around $10^{-47} \mathrm{GeV}^{4}$ [8], which is a small fraction of the energy density up to at least the photon decoupling period. Also the curvature term can be taken as zero, $k=0$, as the universe is observed to be nearly flat. Moreover, under the conditions considered in this paper, the dark radiation term

$$
\chi \propto \frac{1}{a^{4}}
$$

is negligibly small as compared with the other components [24].

An important relation to characterize the matter is the equation of state (EOS) $p_{m}=$ $(\gamma-1) \rho_{m}$. The product particles have different equations of state in various time scales. The index $\gamma$ is $\gamma=4 / 3$ for radiation before the radiation-matter equality time at $t_{e q}=10^{5}$ years $\approx 10^{11}$ sec., and $\gamma \approx 1$ for non-relativistic matter after $t_{e q}$. The constant $w$ determining the EOS of dark matter will be fixed in various scenarios. It takes the value $w=4 / 3$ for hot dark matter $(\mathrm{HDM})$, and $w \approx 1$ for cold dark matter (CDM), just like in the case of ordinary matter.

Next we consider the scalar field properties. In the post-inflationary stage, the inflaton

field executes coherent oscillations about the minimum of the potential [41]. Therefore the kinetic term dominates the potential term in the reheating era, thus leading to

$$
\rho_{\phi}=\frac{\dot{\phi}^{2}}{2}+V(\phi) \approx \frac{\dot{\phi}^{2}}{2} \approx p_{\phi}
$$

The explicit expression of the decay width of the scalar field can be represented as [15],

$$
\Gamma=\alpha_{\phi} M_{\phi} \sqrt{1-\left(\frac{T}{M_{\phi}}\right)^{2}}
$$


where $\alpha_{\phi}$ and $M_{\phi}$ are the coupling constant and the mass of inflaton respectively. Assuming that the thermodynamic equilibrium is established, $T$ is the temperature of the decay product, and it can be related to the matter density $\rho_{m}$. Generally,

$$
\rho_{m}=\sigma T^{\gamma /(\gamma-1)}
$$

where $\sigma$ is a constant. If the matter is in the form of radiation, $\rho_{m}=\pi^{2} T^{4} / 15$, and $\sigma=\pi^{2} / 15$. In the matter-dominated universe, the relation between $\rho_{m}$ and $T$ can also be written down explicitly. The scalar field is negligible small in the matter-dominated phase. In the non-relativistic matter domination era $T \leq 1 \mathrm{eV}$. This is far smaller than the minimum bound obtained for $M_{\phi}[15]$. Hence, in the non-relativistic phases of matter evolution the decay rate is simply $\Gamma=\alpha_{\phi} M_{\phi}$.

Thus, by defining the parameter

$$
\kappa^{2}=\frac{4 \pi G}{3 \lambda},
$$

and with the use of Eqs. (27) - (30), the system of equations describing the reheating process on the brane in the presence of bulk-brane energy exchange is given by

$$
\begin{gathered}
\frac{1}{a} \frac{d a}{d t}=H=\left(\frac{8 \pi G}{3} \rho_{\text {total }}+\kappa^{2} \rho_{\text {total }}^{2}\right)^{\frac{1}{2}} \\
\dot{\rho}_{m}=-3 \gamma H \rho_{m}+2 \alpha_{\phi} M_{\phi} \rho_{\phi} \sqrt{1-\frac{1}{M_{\phi}^{2}}\left(\frac{\rho_{m}}{\sigma}\right)^{2(\gamma-1) / \gamma}}, \\
\dot{\rho}_{\phi}=-6 H \rho_{\phi}-2 \alpha_{\phi} M_{\phi} \rho_{\phi} \sqrt{1-\frac{1}{M_{\phi}^{2}}\left(\frac{\rho_{m}}{\sigma}\right)^{2(\gamma-1) / \gamma}}, \\
\dot{\rho}_{D M}=H\left[\frac{\alpha_{b b}}{2} \rho_{c r}\left(\frac{a_{0}}{a}\right)^{3 w} H-3 w \rho_{D M}\right]
\end{gathered}
$$

In order to simplify the study of the evolution equations during the reheating period, we rescale the time variable and the densities, by introducing a set of dimensionless variables $\left(\tau, r_{m}, r_{D M}, r_{\phi}, r_{c r}\right)$, defined as

$$
t=B \tau, \rho_{m}=A r_{m}, \rho_{D M}=A r_{D M}, \rho_{\phi}=A r_{\phi}, \rho_{c r}=A r_{c r}
$$

where

$$
B=\frac{1}{2 \alpha_{\phi} M_{\phi}},
$$

and

$$
A=\frac{12 \alpha_{\phi}^{2} M_{\phi}^{2}}{8 \pi G}
$$


respectively. By denoting

$$
\eta^{2}=\frac{6 \alpha_{\phi}^{2} M_{\phi}^{2}}{8 \pi G \lambda}, \omega=\frac{1}{M_{\phi}^{2}} \frac{\left(\sqrt{12} \alpha_{\phi} M_{\phi}\right)^{4(\gamma-1) / \gamma}}{(8 \pi G \sigma)^{2(\gamma-1) / \gamma}}, \varepsilon=\alpha_{b b} r_{c r}
$$

the system of equations describing the reheating phase with bulk-brane energy exchange is given by

$$
\begin{gathered}
\frac{1}{a} \frac{d a}{d \tau}=h(\tau)=\sqrt{r_{m}+r_{D M}+r_{\phi}+\eta^{2}\left(r_{m}+r_{D M}+r_{\phi}\right)^{2}} \\
\frac{d r_{m}}{d \tau}=-3 \gamma h(\tau) r_{m}+r_{\phi} \sqrt{1-\omega r_{m}^{2(\gamma-1) / \gamma}} \\
\frac{d r_{\phi}}{d \tau}=-6 h(\tau) r_{\phi}-r_{\phi} \sqrt{1-\omega r_{m}^{2(\gamma-1) / \gamma}} . \\
\frac{d r_{D M}}{d \tau}=h(\tau)\left[\varepsilon\left(\frac{a_{0}}{a}\right)^{3 w}-3 w r_{D M}\right]
\end{gathered}
$$

The system of equations Eqs. (40)-(42) must be considered together with the initial conditions

$$
a(0)=a_{0}, r_{D M}(0)=0, r_{m}(0)=0, r_{\phi}(0)=r_{\phi 0},
$$

where $a_{0}$ and $r_{\phi 0}$ are the initial values of the scale factor and of the scalar field energy density at the beginning of the reheating phase.

In order to obtain a consistent physical interpretation of our results, we need to know the numerical values of the model parameters, which are $\alpha_{\phi}, M_{\phi}$ and $\lambda$, respectively. $\alpha_{\phi}$ and $M_{\phi}$ are not known separately, but the whole quantity $\alpha_{\phi} M_{\phi}$ can be estimated. From Eq.(29), it follows that the order of magnitude of $\alpha_{\phi} M_{\phi}$ is about the order of magnitude of the decay width, which is the reciprocal of the characteristic timescale of reheating. The inflationary era ends, and reheating can start at the earliest at around $t=10^{-32} \mathrm{~s}$, while the Hot Big Bang commences at around $t=10^{-18} \mathrm{~s}$ [40, 41]. The reheating process should complete before the Hot Big Bang to restore the BBN. Therefore $10^{18}$ sec. $^{-1} \leq \Gamma \leq 10^{32}$ sec. ${ }^{-1}$ implies that

$$
1 \mathrm{keV} \leq \alpha_{\phi} M_{\phi} \leq 10^{8} \mathrm{GeV}
$$

As for the brane tension $\lambda$, it is constrained by the validity of Newtonian gravity in 4 dimensions on length scales smaller than $0.1 \mathrm{~mm}$ [42]. The minimum bound is given by [43, 44]

$$
\lambda \geq(100 \mathrm{GeV})^{4} .
$$


With the use of the second transformation in Eqs. (36) we can express the dimensionless matter density $r_{m}$ for an ultra-relativistic gas $(\gamma=4 / 3)$ and with energy-density $\rho_{m}=$ $\pi^{2} T^{4} / 15$ as a function of the temperature $T$ as $r_{m}=4 \pi^{3} G T^{4} / 90 \alpha_{\phi}^{2} M_{\phi}^{2}$. From the definition of the decay width we obtain the constraint $T<M_{\phi}$ on the temperature. Therefore, for a universe in which the normal matter is in the form of radiation only, this condition can be reformulated in a form of a constraint on the dimensionless matter density $r_{m}$, given by

$$
r_{m}<\frac{4 \pi^{3} G M_{\phi}^{2}}{90 \alpha_{\phi}^{2}} .
$$

On the other hand from Eqs. (41) and (42) we obtain the condition $\omega r_{m}^{1 / 2}<1$, which is consistent with Eq. (47).

\section{EXTRA-DIMENSIONAL EFFECTS DOMINATED BRANE REHEATING}

At the end of the inflation, there is no ordinary matter present in the universe. Even in the first early stages of the reheating, the scalar field dominates the matter energy densities, $\rho_{\phi} \gg \rho_{m}, \rho_{D M}$. Due to the high energy of the scalar field, the extra-dimensional effects due to the presence of the fifth dimension are important at this stage,and the quadratic terms in the evolution equations dominate the cosmological dynamics. Therefore one can neglect the linear term in Eq. (40) with respect to the quadratic term. Hence, the extradimensional effects are dominant during the reheating era. Moreover, we assume that the condition $T<<M_{\phi}$ is satisfied during the entire reheating process. This condition implies $\omega r_{m}^{2(\gamma-1) / \gamma}<<1$. Then the equations describing the reheating process with bulk-brane energy transfer are given by

$$
\begin{gathered}
\frac{1}{a} \frac{d a}{d \tau}=\eta\left(r_{m}+r_{D M}+r_{\phi}\right), \\
\frac{d r_{m}}{d \tau}+3 \gamma \frac{1}{a} \frac{d a}{d \tau} r_{m}=r_{\phi}, \\
\frac{d r_{\phi}}{d \tau}+6 \frac{1}{a} \frac{d a}{d \tau} r_{\phi}=-r_{\phi} . \\
\frac{d r_{D M}}{d \tau}+3 w \frac{1}{a} \frac{d a}{d \tau} r_{D M}=\varepsilon\left(\frac{a_{0}}{a}\right)^{3 w} \frac{1}{a} \frac{d a}{d \tau},
\end{gathered}
$$

Eq. (50) can be immediately integrated, and with the use of the initial conditions we obtain the evolution of the energy density of the scalar field during reheating as

$$
r_{\phi}(\tau)=r_{\phi 0}\left(\frac{a_{0}}{a}\right)^{6} e^{-\tau} .
$$


Eq. (51) gives the density of the dark matter on the brane in the form

$$
r_{D M}(\tau)=\varepsilon\left(\frac{a_{0}}{a}\right)^{3 w} \ln \left(\frac{a}{a_{0}}\right) .
$$

The evolution of the matter energy density may be represented as

$$
r_{m}(\tau)=r_{\phi 0} a_{0}^{6-3 \gamma}\left(\frac{a_{0}}{a}\right)^{3 \gamma} \int_{0}^{\tau} a^{3 \gamma-6} e^{-\tau} d \tau .
$$

\section{A. The stiff matter case}

The value $\gamma=2$ corresponds to the case of the so-called stiff matter fluid, with equation of state given by $p_{m}=\rho_{m}$. In this case the energy density of the matter is given by

$$
r_{m}(\tau)=r_{\phi 0}\left(1-e^{-\tau}\right)\left(\frac{a_{0}}{a}\right)^{6}, \gamma=2 .
$$

The sum of the energy densities of the matter and scalar field satisfies the equation

$$
\frac{d}{d \tau}\left(r_{m}+r_{\phi}\right)=-\left(3 \gamma r_{m}+6 r_{\phi}\right) \frac{1}{a} \frac{d a}{d \tau} .
$$

For $\gamma=2$ we obtain

$$
r_{m}+r_{\phi}=r_{\phi 0}\left(\frac{a_{0}}{a}\right)^{6} .
$$

The scale factor of the universe can be obtained from the general equation

$$
\begin{aligned}
\frac{1}{a} \frac{d a}{d \tau}= & \eta\left\{r_{\phi 0} a_{0}^{6-3 \gamma}\left(\frac{a_{0}}{a}\right)^{3 \gamma}\left[\int a^{3 \gamma-6} e^{-\tau} d \tau-\beta\left(a_{0}\right)\right]+\right. \\
& \left.\varepsilon\left(\frac{a_{0}}{a}\right)^{3 w} \ln \left(\frac{a}{a_{0}}\right)+r_{\phi 0}\left(\frac{a_{0}}{a}\right)^{6} e^{-\tau}\right\} .
\end{aligned}
$$

In the case in which both the dark matter and the usual matter are in the ultra-relativistic state, $\gamma=w=2$, and the equation describing the scale factor dynamics is given by

$$
a^{5} \frac{d a}{d \tau}=\eta a_{0}^{6}\left[r_{\phi 0}+\varepsilon \ln \left(\frac{a}{a_{0}}\right)\right], \gamma=w=2 .
$$

In this case the time evolution of the universe during the extra-dimensions dominated phase of the reheating era can be expressed as

$$
\tau=\frac{1}{\varepsilon \eta} \exp \left(-\frac{6 r_{\phi 0}}{\varepsilon}\right) \operatorname{Ei}\left[\frac{6 r_{\phi 0}}{\varepsilon}+6 \ln \left(\frac{a}{a_{0}}\right)\right],
$$

where $\operatorname{Ei}(z)=-\int_{-z}^{\infty} e^{-t} d t / t$ is the exponential integral function, and where the principal value of the integral must be taken. In a power series representation the dimensionless time parameter $\tau$ can be obtained in terms of the scale factor $a$ as

$$
\tau \approx \frac{1}{\varepsilon \eta}\left(\frac{a}{a_{0}}\right)^{6}\left\{\frac{1}{6 r_{\phi 0} / \varepsilon+6 \ln \left(a / a_{0}\right)}+\left[\frac{1}{6 r_{\phi 0} / \varepsilon+6 \ln \left(a / a_{0}\right)}\right]^{2}+\ldots\right\} .
$$




\section{B. The radiation dominated phase}

In order to obtain a clear physical picture of the reheating process during the extradimensional effects dominated era one must study the evolution equations Eqs. (48) - (51) numerically. In order to do this we assume that the normal matter is always generated from the inflaton field in the form of ultra-relativistic particles, leading to a very hot and high density initial state of the universe, an assumption which is consistent with the standard hot Big-Bang model. Therefore we take $\gamma=4 / 3$. As for the dark matter, it can be injected from the bulk both as a relativistic fluid, with $w=4 / 3$, or as a non-relativistic, pressureless fluid, with $w=1$. The variation of the radiation energy density is represented, for both equations of state of the dark matter, for fixed values of the parameters $\varepsilon$ and $\omega$ and for different values of the parameter $\eta$ in Fig. 1.
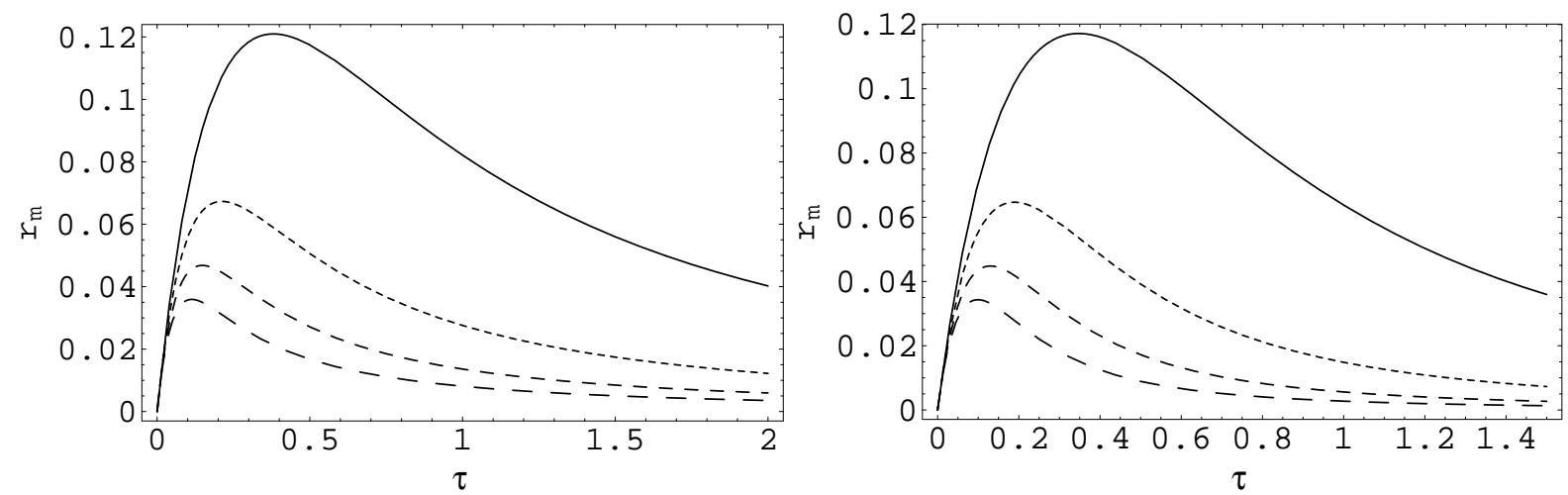

FIG. 1: Variation of the energy density of the radiation $(\gamma=4 / 3)$ during the extra-dimensional effects dominated reheating period, with ultra-relativistic dark matter injection from the bulk $(w=4 / 3)$ (figure on the left) and non-relativistic pressureless dark matter injection $(w=1)$ (figure on the right), respectively, for $\omega=0.5, \varepsilon=6$ and different values of $\eta: \eta=0.5$ (solid curve), $\eta=1$ (dotted curve), $\eta=1.5$ (short dashed curve) and $\eta=2$ (long dashed curve). The initial values of the scalar field density and of the scale factor are $r_{\phi 0}=1$ and $a_{0}=10^{-3}$, respectively.

The variation of the dark matter energy density is represented in Fig. 2.

The variations of the scalar field energy densities and of the scale factors in these two situations are represented in Figs. 3 and 4, respectively.

As one can see from the Figs. 1 and 2, during the reheating period the energy of the scalar field is transferred to the normal matter (radiation). The energy density of the 

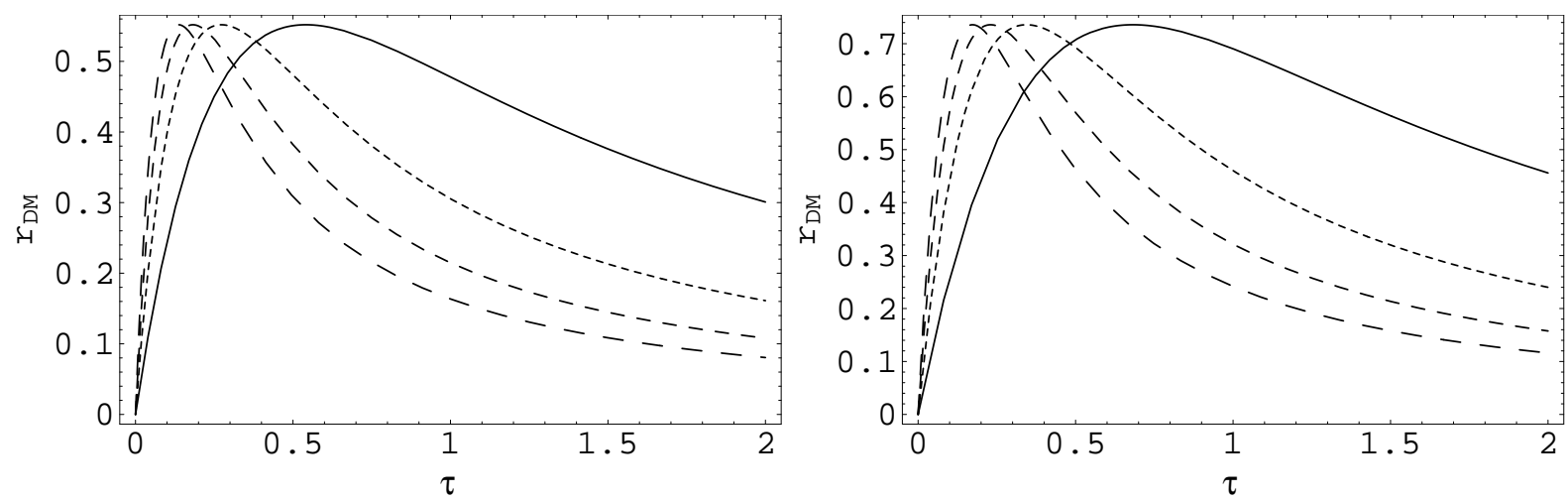

FIG. 2: Variation of the energy density of the ultra-relativistic dark matter $(w=4 / 3)$ (figure on the left) and of the pressureless non-relativistic dark matter $(w=1)$ (figure on the right), respectively, during the extra-dimensional effects dominated reheating period, for $\omega=0.5, \varepsilon=6$ and different values of $\eta: \eta=0.5$ (solid curve), $\eta=1$ (dotted curve), $\eta=1.5$ (short dashed curve) and $\eta=2$ (long dashed curve). The initial values of the scalar field density and of the scale factor are $r_{\phi 0}=1$ and $a_{0}=10^{-3}$, respectively. The normal matter is in the form of radiation with $\gamma=4 / 3$.
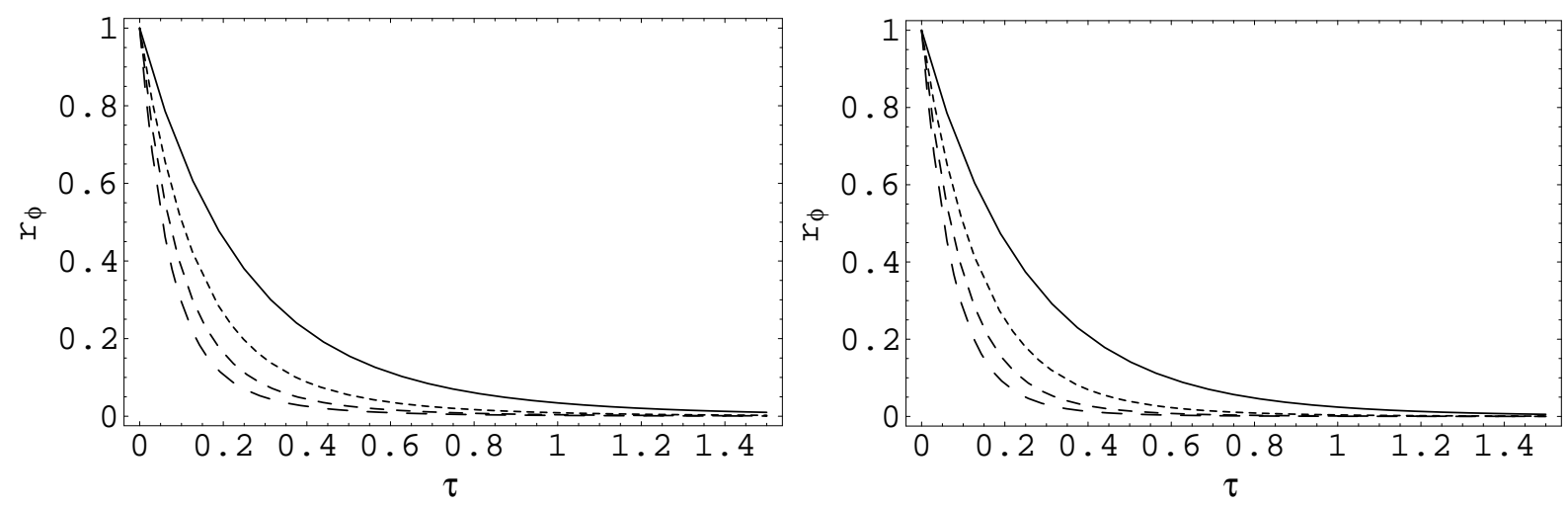

FIG. 3: Time variation of the energy density of the inflaton field during the extra-dimensional effects dominated reheating period, with ultra-relativistic dark matter injection from the bulk $(w=4 / 3)$ (figure on the left) and non-relativistic pressureless dark matter injection $(w=1)$ (figure on the right), respectively, for $\omega=0.5, \varepsilon=6$ and different values of $\eta$ : $\eta=0.5$ (solid curve), $\eta=1$ (dotted curve), $\eta=1.5$ (short dashed curve) and $\eta=2$ (long dashed curve). The initial values of the scalar field density and of the scale factor are $r_{\phi 0}=1$ and $a_{0}=10^{-3}$, respectively. The normal matter is in the form of radiation with $\gamma=4 / 3$.

matter increases from zero to a maximum value, and, after reaching the maximum, it starts to decrease, due to the cosmological expansion. During the same period there is an injection 

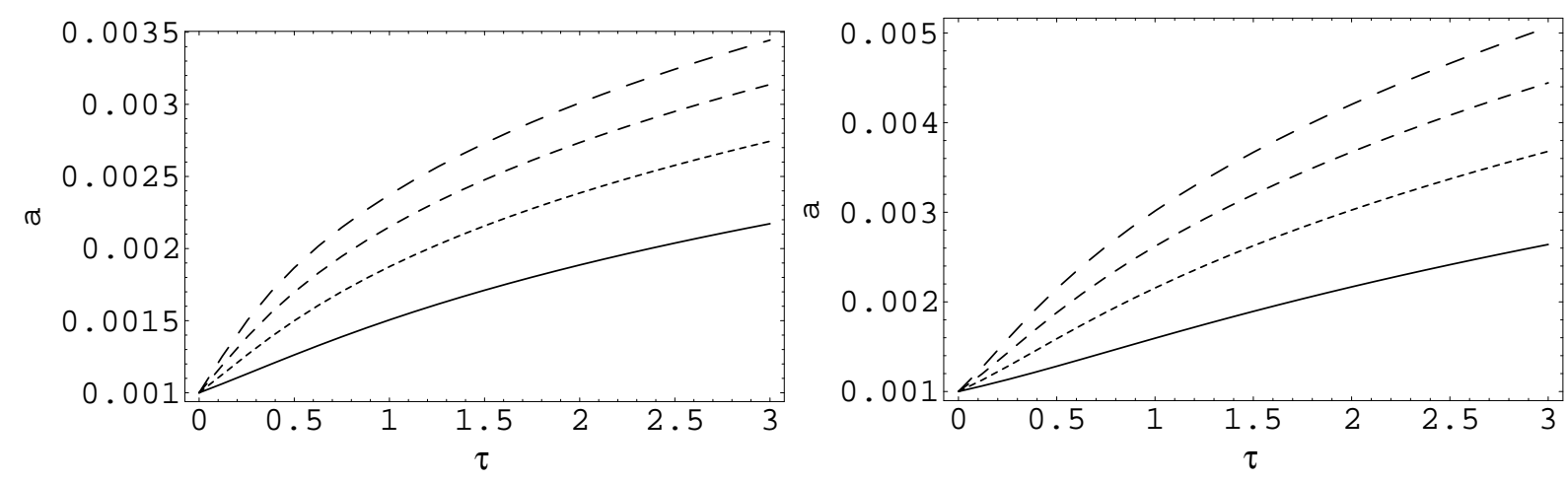

FIG. 4: Time variation of the scale factor of the universe during the extra-dimensional effects dominated reheating period, with ultra-relativistic dark matter injection from the bulk $(w=4 / 3)$ (figure on the left) and non-relativistic pressureless dark matter injection $(w=1)$ (figure on the right), respectively, for $\omega=0.5, \varepsilon=6$ and different values of $\eta: \eta=0.5$ (solid curve), $\eta=1$ (dotted curve), $\eta=1.5$ (short dashed curve) and $\eta=2$ (long dashed curve). The initial values of the scalar field density and of the scale factor are $r_{\phi 0}=1$ and $a_{0}=10^{-3}$, respectively. The normal matter is in the form of radiation with $\gamma=4 / 3$.

of dark matter from the bulk, the energy density of the dark matter increasing from an initial zero value to a maximum value, which depends on the equation of state of the dark matter and on the parameter $\eta$, describing the magnitude of the extra-dimensional effects. During the reheating process the energy density of the scalar field decreases to zero.

\section{GENERAL EVOLUTION OF THE BRANE UNIVERSE DURING THE RE- HEATING PERIOD}

After reaching its maximum value during the reheating period, due to the cosmological expansion, the energy density of the matter (in all its forms) decreases in time and for time intervals $t>t_{c r}$ the condition $\rho_{\text {total }} / \lambda<<1$ is satisfied. In this case the quadratic contributions to the field equations can be neglected, and the universe is described by the standard general relativistic cosmological models. In the dimensionless formulation of the field equations the general relativistic limit corresponds to $\eta \rightarrow \infty$. In the general case, when all terms are considered in the field equations, the evolutions of the matter and of the scalar field is given generally by Eqs. (52) - (54), respectively. However, the linear term in energy density modifies the expansion rate of the universe, which is described by Eq. (40). 
From the mathematical structure of the field equations one can derive some general properties of the model, and of the cosmological evolution during the reheating. The normal matter and the dark matter reach a maximum value at a moment $\tau=\tau_{\max }$, the maximum values of the densities being given by the solutions of the equations $d r_{m} / d \tau=0$ and $r_{D M} / d \tau=0$, respectively. In the case of the dark matter the maximum value is given by

$$
r_{D M}^{(\max )}=\left.\frac{\varepsilon}{3 w}\left(\frac{a_{0}}{a}\right)^{3 w}\right|_{\tau=\tau_{\max }} .
$$

The maximum value of the dark matter density is determined by the injection rate $\varepsilon$, the cosmological dynamics, determining value of the scale factor at $\tau_{\max }$, and by the equation of state of the dark matter. By assuming that $\omega r_{m}^{2(\gamma-1) / \gamma}<<1$, the maximum value of the normal matter density can be obtained from the algebraic equation

$$
r_{m}^{(\max )}=\left.\frac{r_{\phi 0}}{3 \gamma h(\tau)}\left(\frac{a_{0}}{a}\right)^{6} e^{-\tau}\right|_{\tau=\tau_{\max }} .
$$

In the case of the extra-dimensional effects dominated evolution, $h(\tau)=$ $\eta\left(r_{m}+r_{D M}+r_{\phi}\right)$, and Eq. (63) gives

$$
r_{m}^{(\max )}=\frac{r_{\phi}\left(\tau_{\max }\right)+r_{D M}^{(\max )}}{2}\left\{\sqrt{1+\frac{4 r_{\phi}\left(\tau_{\max }\right)}{3 \gamma \eta\left[r_{\phi}\left(\tau_{\max }\right)+r_{D M}^{(\max )}\right]^{2}}}-1\right\}
$$

which can be approximated as

$$
r_{m}^{(\max )} \approx \frac{r_{\phi}\left(\tau_{\max }\right)}{3 \gamma \eta\left[r_{\phi}\left(\tau_{\max }\right)+r_{D M}^{(\max )}\right]}=\frac{1}{3 \gamma \eta}\left[1+\frac{r_{D M}^{(\max )}}{r_{\phi}\left(\tau_{\max }\right)}\right]
$$

The maximum value of the matter energy density is inverse proportional to the parameter $\eta=\sqrt{3 / 4 \pi G \lambda} \alpha_{\phi} M_{\phi}$. In the case of a fixed value of $\alpha_{\phi} M_{\phi}$, a large value of $\eta$, corresponding, for example, to a small brane tension $\lambda$, would determine a decrease of the maximum matter density, while small values of $\eta$ (large brane tensions) would increase the amount of matter produced during the reheating phase. In terms of the scale factor the maximum matter density can be expressed as

$$
r_{m}^{(\max )} \approx \frac{1}{3 \gamma \eta}\left\{1+\frac{\varepsilon}{3 w r_{\phi 0}}\left[\frac{a_{0}}{a\left(\tau_{\max }\right)}\right]^{3 w-6} e^{\tau_{\max }}\right\} .
$$

Generally, the ratio $u$ of the dark matter and normal matter energy density can be represented, as a function of the scale factor, as

$$
u=\frac{r_{D M}(\tau)}{r_{m}(\tau)}=\frac{\varepsilon}{r_{\phi 0}} a_{0}^{3 \gamma-6}\left(\frac{a_{0}}{a}\right)^{3 w-3 \gamma} \frac{\ln \left(a / a_{0}\right)}{\int_{0}^{\tau} a^{3 \gamma-6} e^{-\tau} d \tau} .
$$


In the small time limit the energy density of the baryonic and of the dark matter is negligibly small with respect to the energy density of the scalar field, which in the very early stages of the reheating is given by Eq. (52). Therefore in the limit of $\tau<<1$, the evolution of the scale factor is obtained as $a \approx a_{0}\left(6 \eta r_{\phi 0}\right)^{1 / 6} \tau^{1 / 6}$. Hence the small time behavior of $u$ is described by the equation

$$
u \approx \frac{\epsilon(6 \eta)^{1 / 6}}{4 r_{\phi 0}^{2 / 3}} \frac{\ln \left(6 \eta r_{\phi 0} \tau\right)}{\tau^{2 / 3}}, \tau<<1,
$$

Since in the limit of large time the function $a^{3 \gamma-6} e^{-\tau}$ tends to zero, it follows that the value of the integral $\int_{0}^{\tau} a^{3 \gamma-6} e^{-\tau} d \tau$ can be written as a function of the initial value of the scale factor $a_{0}$, so that $\int_{0}^{\tau} a^{3 \gamma-6} e^{-\tau} d \tau \approx \beta\left(a_{0}\right)$.

Therefore in the large time limit the ratio of the densities of the dark matter and of the baryonic matter can be written as

$$
u=\frac{\varepsilon a_{0}^{3 \gamma-6}}{r_{\phi 0} \beta\left(a_{0}\right)}\left(\frac{a_{0}}{a}\right)^{3 w-3 \gamma} \ln \left(\frac{a}{a_{0}}\right), \tau>>1 .
$$

Precision cosmological observations have shown that $u$ has a constant numerical value, at a high degree of accuracy. Since in the present day universe the baryonic matter is non-relativistic, with $\gamma=1$, the constancy of $u$ requires for the dark matter to also be in a non-relativistic (cold) phase, with $w=1$. The magnitude of the dark matter/baryonic matter ratio depends on the initial conditions of the universe during the reheating period, namely, the dark matter injection rate, the energy density of the scalar field and the value of the scale factor. On the other hand, the ratio of the densities of the dark matter and of the normal matter is not an absolute constant during the cosmological evolution, but it is a very slowly, logarithmically increasing function of time.

The results of the numerical integration of the Eqs. (40) - (43) are represented in Figs. 5-8, respectively. Fig. 5 presents the variation of the normal matter energy density during reheating, with the linear terms in the density also included, for the two equations of state of the dark matter. The variation of the dark matter energy density is represented in Fig. 6.

The variations of the scalar field energy densities and of the scale factors for the two equations of state are represented in Figs. 7 and 8, respectively.

As one can see from the figures, the inclusion of the linear matter terms into the evolution equations leads to an overall decrease of the numerical values of the cosmological parame- 

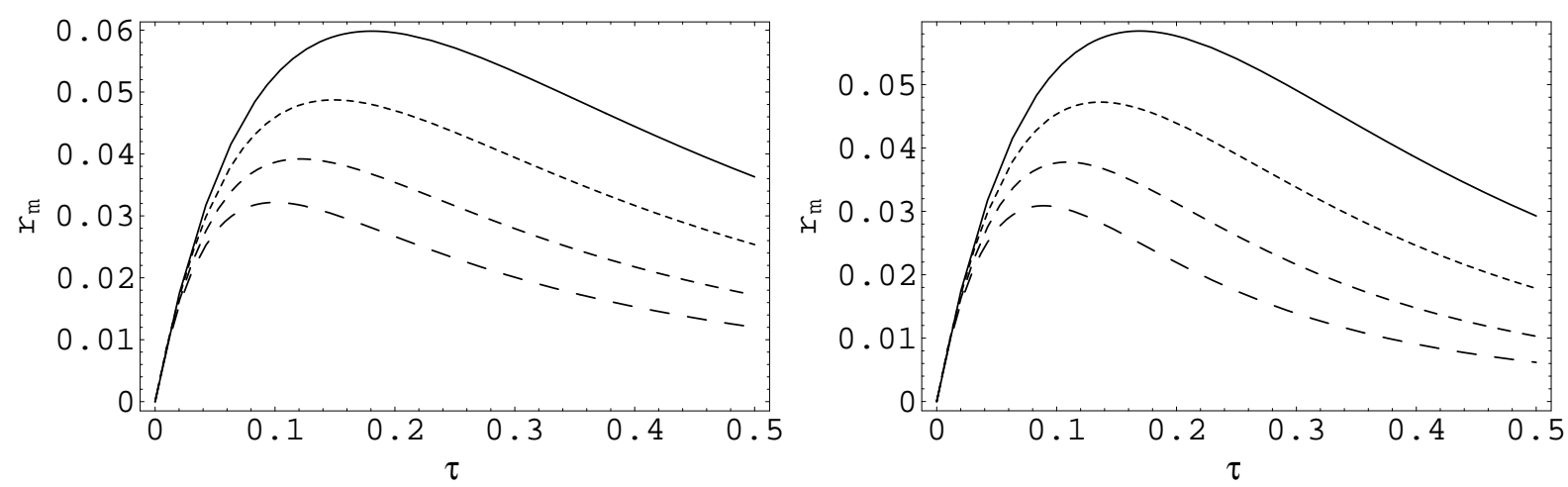

FIG. 5: Variation of the energy density of the photon gas $(\gamma=4 / 3)$ during the reheating period, with ultra-relativistic dark matter injection from the bulk $(w=4 / 3)$ (figure on the left) and non-relativistic pressureless dark matter injection $(w=1)$ (figure on the right), respectively, for $\omega=0.5, \varepsilon=6$ and different values of $\eta: \eta=0.5$ (solid curve), $\eta=1$ (dotted curve), $\eta=1.5$ (short dashed curve) and $\eta=2$ (long dashed curve). The initial values of the scalar field density and of the scale factor are $r_{\phi 0}=1$ and $a_{0}=10^{-3}$, respectively.
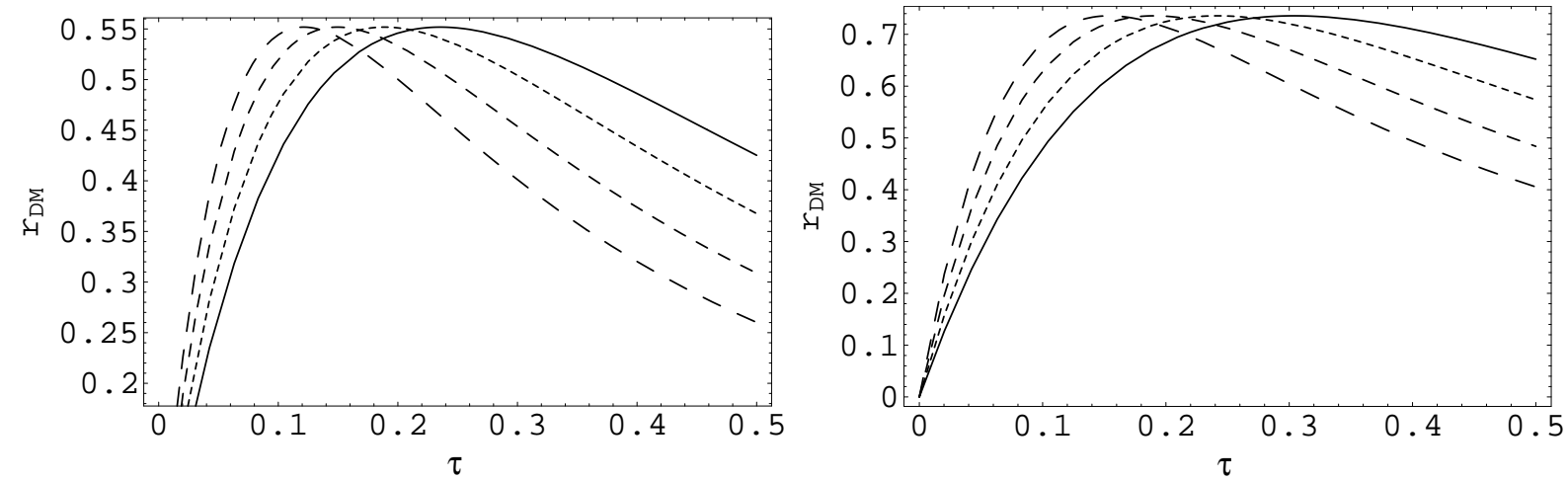

FIG. 6: Variation of the energy density of the ultra-relativistic dark matter $(w=4 / 3)$ (figure on the left) and of the pressureless non-relativistic dark matter $(w=1)$ (figure on the right) during the reheating period, for $\omega=0.5, \varepsilon=6$ and different values of $\eta: \eta=0.5$ (solid curve), $\eta=1$ (dotted curve), $\eta=1.5$ (short dashed curve) and $\eta=2$ (long dashed curve). The initial values of the scalar field density and of the scale factor are $r_{\phi 0}=1$ and $a_{0}=10^{-3}$, respectively. The normal matter is in the form of radiation with $\gamma=4 / 3$.

ters during the reheating period. If in the case of the extra-dimensional effects dominated dynamics the maximum values of the matter energy density are of the orders of $r_{m} \approx 0.12$, in the general case and for the same value of the physical parameters this value is $r_{m} \approx 0.06$. 

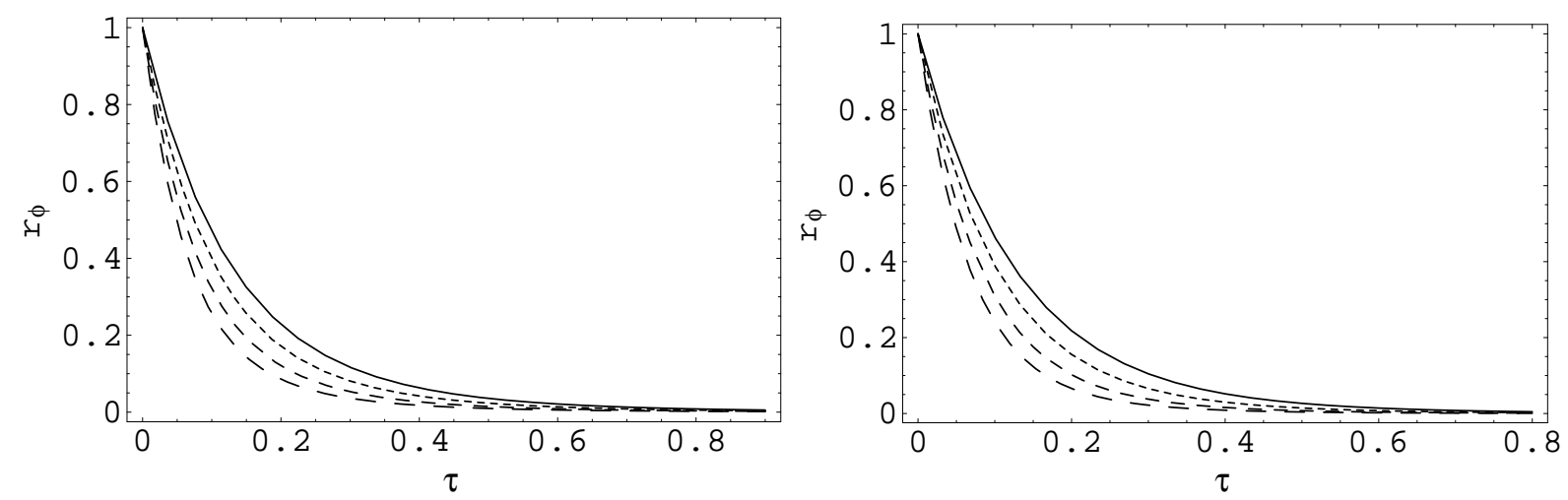

FIG. 7: Time variation of the energy density of the inflaton field during the reheating period, with ultra-relativistic dark matter injection from the bulk $(w=4 / 3)$ (figure on the left) and non-relativistic pressureless dark matter injection $(w=1)$ (figure on the right), respectively, for $\omega=0.5, \varepsilon=6$ and different values of $\eta: \eta=0.5$ (solid curve), $\eta=1$ (dotted curve), $\eta=1.5$ (short dashed curve) and $\eta=2$ (long dashed curve). The initial values of the scalar field density and of the scale factor are $r_{\phi 0}=1$ and $a_{0}=10^{-3}$, respectively. The normal matter is in the form of radiation with $\gamma=4 / 3$.
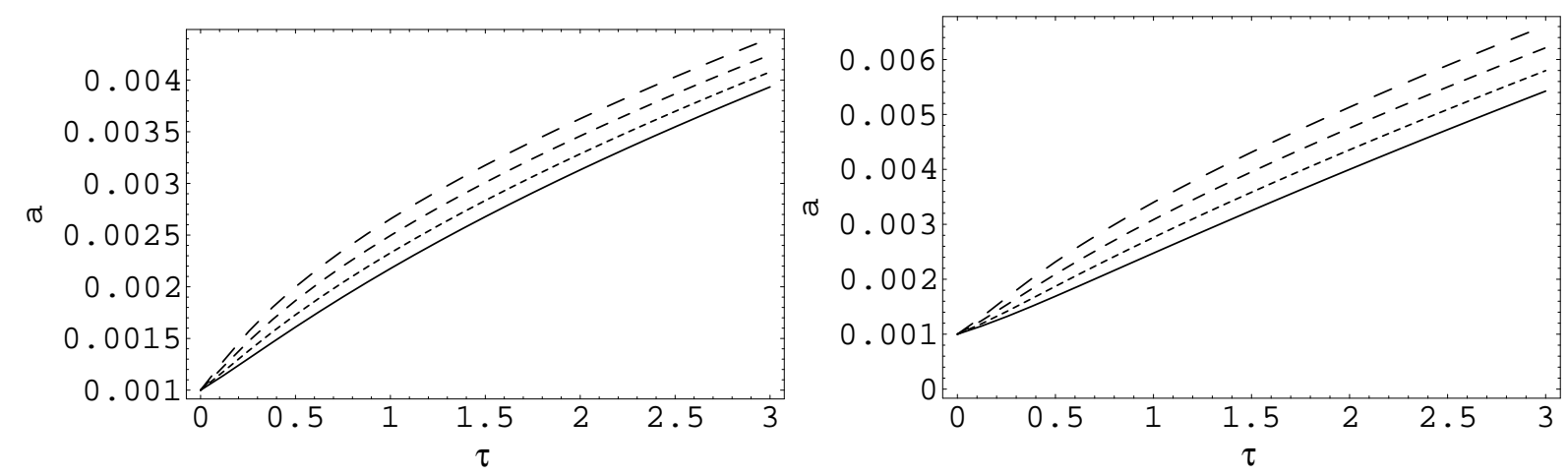

FIG. 8: Time variation of the scale factor of the universe during the reheating period, with ultrarelativistic dark matter injection from the bulk $(w=4 / 3)$ (figure on the left) and non-relativistic pressureless dark matter injection $(w=1)$ (figure on the right), respectively, for $\omega=0.5, \varepsilon=6$ and different values of $\eta: \eta=0.5$ (solid curve), $\eta=1$ (dotted curve), $\eta=1.5$ (short dashed curve) and $\eta=2$ (long dashed curve). The initial values of the scalar field density and of the scale factor are $r_{\phi 0}=1$ and $a_{0}=10^{-3}$, respectively. The normal matter is in the form of radiation with $\gamma=4 / 3$.

However, the maximum value of the energy density of the dark matter is not significantly influenced by the change in the cosmological dynamics, since it is essentially determined by 
the injection process from the bulk. On the other hand the decay of the inflaton field is more rapid when the full dynamics on the brane is taken into account.

It is easy to show that the ratio of the dark matter and normal matter energy density $u$ satisfies the following evolution equation

$$
\frac{d u}{d \tau}=\left[(3 \gamma-3 w) h(\tau)-\frac{r_{\phi}}{r_{m}} \sqrt{1-\omega r_{m}^{2(\gamma-1) / \gamma}}\right] u+\varepsilon\left(\frac{a_{0}}{a}\right)^{3 w} \frac{h(\tau)}{r_{m}} .
$$

The maximum value of $u, u_{\max }=u\left(\tau_{\max }\right)$ is given by the solution of the equation $d u /\left.d \tau\right|_{\tau=\tau_{\max }}=0$, giving for the maximum value of $u$ the expression

$$
u_{\max }=\left.\frac{\varepsilon\left(a_{0} / a\right)^{3 w} h(\tau)}{r_{\phi} \sqrt{1-\omega r_{m}^{2(\gamma-1) / \gamma}}-(3 \gamma-3 w) h(\tau) r_{m}}\right|_{\tau=\tau_{\max }}
$$

In the limit of large times, when $r_{\phi} \approx 0$, and assuming that the dark matter obeys the same equation of state as the normal matter, $w=\gamma$, $u$ satisfies the equation

$$
\frac{d u}{d \tau}=\varepsilon\left(\frac{a_{0}}{a}\right)^{3 w} \frac{h(\tau)}{r_{m}}
$$

For non-relativistic dark matter and normal matter we obtain the simple relation

$$
\frac{d u}{d \tau}=\varepsilon h(\tau)
$$

Thus the variation of the ratio of the dark matter and of the normal matter is proportional to the Hubble parameter.

To study the time variation of the ratio of the dark and baryonic matter $u$ we consider that the transition between the relativistic and non-relativistic state can be modelled as a smooth transition, with the equations of state of the form $p_{D M}=w(t) \rho_{D M}$ and $p_{m}=\gamma(t) \rho_{m}$, respectively. In the dimensionless time variable $\tau$ we assume that the functions $\gamma(\tau)$ and $w(\tau)$ can be given as

$$
\gamma(\tau)=\frac{4}{3} \frac{\tau_{o \gamma}}{\tau+\tau_{0 \gamma}}+\frac{\tau}{\tau+\tau_{0 \gamma}}
$$

and

$$
w(\tau)=\frac{4}{3} \frac{\tau_{o w}}{\tau+\tau_{0 w}}+\frac{\tau}{\tau+\tau_{0 w}}
$$

respectively, where $\tau_{o \gamma}$ and $\tau_{o w}$ are the critical transitions times for the dark matter and the baryonic matter. When $\tau<<\tau_{0 \gamma}, \tau_{0 w}, \gamma \approx 4 / 3$, while for $\tau>>\tau_{0 \gamma}, \tau_{0 w}, \gamma \approx 1$. The time variation of $u$ is represented, for two different numerical values of the critical transitions times of the dark matter and baryonic matter, respectively, and for different values of the physical parameters, in Fig. 9. 


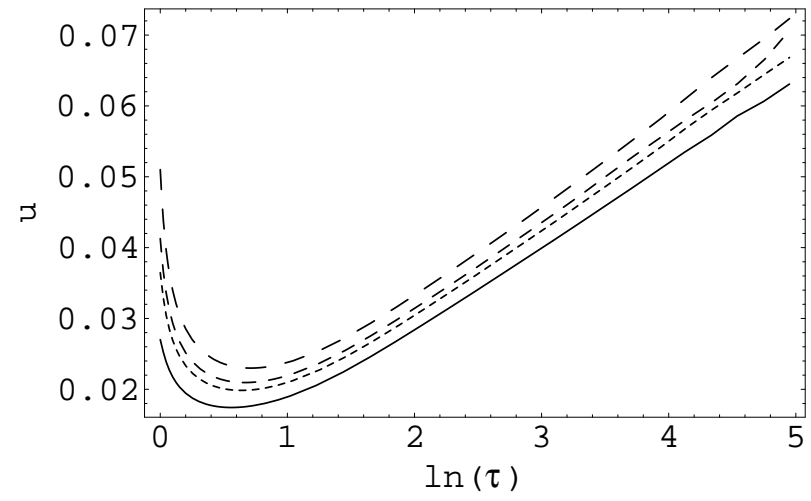

FIG. 9: Time variation of the ratio $u$ of the dark matter and normal matter energy densities during the reheating period, by assuming a smooth transition from the relativistic to the non-relativistic state, so that $\gamma(\tau)=(4 / 3) \tau_{o \gamma} /\left(\tau+\tau_{0 \gamma}\right)+\tau /\left(\tau+\tau_{0 \gamma}\right)$ and $w(\tau)=(4 / 3) \tau_{o w} /\left(\tau+\tau_{0 w}\right) \tau /\left(\tau+\tau_{0 w}\right)$, respectively. The values of the parameters are $\omega=0.5, \eta=2$ (solid curve), $\eta=2.5$ (dotted curve), $\eta=3$ (short dashed curve) and $\eta=3.5$ (long dashed curve). We have assumed that the transitions from a non-relativistic to a relativistic phase takes place at the critical time $\tau_{0 \gamma}=\tau_{0 w}=10^{8}$. The initial values of the scalar field density and of the scale factor are $r_{\phi 0}=1$ and $a_{0}=10^{-3}$, respectively, while the dark matter injection rate is $\varepsilon=0.01$.

\section{DISCUSSIONS AND FINAL REMARKS}

In the present paper we have considered the reheating of the universe in the brane world models with bulk-brane energy exchange. Our model is essentially based on the hypothesis that the present day dark matter, representing around $25 \%$ of the total matter content of the universe, was transferred onto the brane during the post-inflationary reheating period, while the normal matter is the result of the decay of the inflaton field into a ultra-relativistic photon gas. Hence, after the inflationary epoch nor dark matter neither normal matter were present on the brane, and they were created during the reheating.

To study the reheating process we have introduced a set of dimensionless quantities, which describe the evolution of the scaled densities in terms of a dimensionless time parameter $\tau$. By using the results of the numerical simulations for the rescaled variables one can obtain some constraints on the physical parameters of the model.

The reheating time $t_{r e h}$, the time after which the universe is reheated to a temperature allowing the standard hot big bang model to take place is related to the dimensionless time 
$\tau_{\text {reh }}$ by Eqs. (36) and (37), respectively. These equations allows us to constrain the mass and the coupling constant of the inflaton field as

$$
\alpha_{\phi} M_{\phi}=\frac{\tau_{r e h}}{2 t_{r e h}}=0.328 \tau_{r e h} t_{r e h}(\mathrm{~s}) \times 10^{-24} \mathrm{GeV}
$$

Since according the hot standard big-bang scenario reheating must end at around $t_{r e h}=$ $10^{-18} \mathrm{~s}$, it follows that $\alpha_{\phi} M_{\phi}=0.328 \tau_{r e h} \mathrm{keV}$. In our model and for the typical values of the physical parameters we have adopted (bulk-brane injection rate and brane tension), according to the numerical analysis reheating may definitely take place for values of $\tau_{r e h}$ in the range $0.1 \leq \tau_{r e h} \leq 1$ (see Figs. 1-2 and 5-6), thus giving $0.0328 \mathrm{keV} \leq \alpha_{\phi} M_{\phi} \leq$ $0.328 \mathrm{keV}$. Values of $\alpha_{\phi} M_{\phi}$ of the order of $1 \mathrm{keV}$ or higher would require values of $\tau_{r e h}$ of the order of $\tau_{\text {reh }} \geq 3$.

The numerical analysis of the field equations, as well as their qualitative study, shows the existence of an absolute maximum value of the matter density $\rho_{\text {matter }}^{(\max )}$ during the cosmological evolution of the universe. The maximum value of the total matter density is given by

$$
\rho_{\text {matter }}^{(\max )}=\left[r_{m}^{(\max )}+r_{D M}^{(\max )}\right] \frac{12 \alpha_{\phi}^{2} M_{\phi}^{2}}{8 \pi G}=7.13\left[r_{m}^{(\max )}+r_{D M}^{(\max )}\right]\left(\alpha_{\phi} M_{\phi} / \mathrm{GeV}\right)^{2} \times 10^{37} \mathrm{GeV}^{4} .
$$

By assuming that $r_{m}^{(\max )}+r_{D M}^{(\max )} \approx 0.5-0.7$ and $\alpha_{\phi} M_{\phi} \approx 1 \mathrm{keV}$, we obtain

$$
3.565 \times 10^{25} \mathrm{GeV}^{4} \leq \rho_{\text {matter }}^{(\max )} \leq 4.991 \times 10^{25} \mathrm{GeV}^{4}
$$

Eq. (78) allows the determination of the reheating temperature $T_{r e h}$ of the universe, once the equation of state of the matter is known. Assuming that both normal matter and dark matter are in a ultra-relativistic state, we obtain

$$
T_{r e h} \approx 3 \times 10^{6} \mathrm{GeV}
$$

Several estimations of the numerical value of the reheating temperature have been obtained by using some observational constraints. A strong constraint on the cosmological models comes from the study of the CMB anisotropy, and requires that the results do not exceed the observed level of normalized anisotropy on large scales [10, 15]. By also requiring that the radiative corrections mediated by the couplings do not spoil the flatness of the potential limits, it follows that the reheating temperature must be below the grand unification theory scale, $T_{r e h}<10^{16} \mathrm{GeV}$, which physically means that the grand unified 
theory symmetries are not restored and therefore there is no second phase of production of monopoles [10, 15]. By assuming that the energy density of the universe is in the form of relativistic matter with density $\rho_{\text {total }}=g_{*} \pi^{2} T^{4} / 30$, where $g_{*}$ is the effective number of massless degrees of freedom $\left(g_{*}=100-1000\right)$, the reheating temperature can be obtained as $T_{r e h} \approx 0.2\left(100 / g_{*}\right)^{1 / 4} \sqrt{\Gamma_{t o t} m_{P l}} \approx 0.2\left(100 / g_{*}\right)^{1 / 4} \alpha_{\phi}^{1 / 2} \sqrt{M_{\phi} m_{P l}}$, where $\Gamma_{\text {tot }}$ is the total decay width and $m_{P l}$ is the Planck mass. From the study of the CMB anisotropies it follows that $M_{\phi} \approx 10^{-6} m_{P l}[10,15]$.

In the context of supersymmetry models, a stronger constraint may be obtained from the production rate of gravitinos, the supersymmetric partners of the gravitons. The gravitinos, having a long lifetime, of the order of $10^{5} \mathrm{~s}$, may cause serious problems in cosmology. For a wide range of the gravitino masses in order to obtain consistency with the nucleosynthesis constraints, the reheating temperature must be below $10^{9} \mathrm{GeV}, T_{\text {reh }}<10^{9} \mathrm{GeV}[10,30]$. A stronger constraint on the reheating temperature for the gravitino decay products not to destroy the light elements synthesized during the Big-Bang nucleosynthesis was obtained in [45], and requires that $T_{r e h}$ must satisfy the condition $T_{r e h} \leq 10^{6}-10^{7} \mathrm{GeV}$. Primordial nucleosynthesis requires that the universe is close to thermal equilibrium at a temperature of around 1-4 MeV [11]. Hence, the reheating temperature predicted by our model satisfies all the CMB and nucleosynthesis constraints.

By adopting the $\mathrm{CMB}$ constraint implying that $M_{\phi} \approx 10^{-6} m_{P l} \approx 1.22 \times 10^{19} \mathrm{GeV}$, we can estimate the inflaton field coupling constant as $\alpha_{\phi} M_{\phi}=0.328 \tau_{\text {reh }} t_{r e h}(s) \times 10^{-24} / M_{\phi}=$ $2.69 \times \tau_{r e h} t_{r e h}(s) \times 10^{-44}$. For $t_{r e h} \approx 10^{-18} \mathrm{~s}$ we obtain $\alpha_{\phi} \approx 2.69 \times \tau_{r e h} \times 10^{-26}$, or $2.69 \times 10^{-27} \leq \alpha_{\phi} \leq 2.69 \times 10^{-26}$.

The numerical values of the cosmological parameters sensitively depend on the parameter $\eta$, a dimensionless combination of the mass and coupling constant of the inflaton field and of the brane tension, respectively. From Eq. (38) we obtain the brane tension $\lambda$ as

$$
\lambda=\frac{6 \alpha_{\phi}^{2} M_{\phi}^{2}}{8 \pi G \eta^{2}}=\frac{3.565}{\eta^{2}}\left(\alpha_{\phi} M_{\phi} / \mathrm{GeV}\right)^{2} \times 10^{37} \mathrm{GeV}^{4}
$$

Since from the results of the numerical integration it follows that the values of $\eta$ are of the order of $0.5 \leq \eta \leq 2$, and by adopting again for $\alpha_{\phi} M_{\phi}$ a value of $1 \mathrm{keV}$, we obtain $0.891 \times 10^{25} \mathrm{GeV}^{4} \leq \lambda \leq 14.26 \times 10^{25} \mathrm{GeV}^{4}$. This value is much larger than the minimal value $\lambda \geq 10^{8} \mathrm{GeV}^{4}$ previously obtained in [43, 44], but it is consistent with the bound $\lambda \leq 10^{40} \mathrm{GeV}^{4}$ derived in [46]. 
In the present model the reheating temperature satisfies with a very good approximation the relation

$$
T_{\text {reh }}=\lambda^{1 / 4},
$$

relating the reheating temperature to the brane tension, and which was first suggested in [47]. The universe exits the extra-dimensions dominated phase, when $\rho_{\text {total }}>\lambda$, at a density $\rho_{\text {total }} \approx \lambda$, which, assuming that the matter and field content is formed from ultrarelativistic particles, gives a transition temperature $T_{\text {trans }} \sim \lambda^{1 / 4}$. According to our results, $T_{\text {trans }} \approx T_{\text {reh }}$. Therefore, the reheating phase of the universe essentially took place during the extra-dimensions dominated cosmological era, when the bulk effects may have played a major role in the dynamics and cosmological defrosting of the universe. On the other hand, since the reheating temperature may be estimated from observations, the exact relation between $T_{r e h}$ and $\lambda$ gives the possibility of the direct determination of the brane tension from cosmological observations.

The basic feature of the present reheating model is the continuous energy-matter transfer between the brane and the bulk. Even that this transfer was maximum during the reheating period, essentially contributing in establishing the present day cosmological chemical composition of the universe, a very small rate of change of the ratio of the dark matter and of the normal matter still occurred during the later phases of the cosmological evolution. The present day rate of change of this ratio can be estimated from Eq. (73), and is given by

$$
\frac{d u}{d t} \approx \varepsilon \times H_{0} \approx 3.24 \varepsilon \times 10^{-18} \mathrm{~s}^{-1},
$$

where $H_{0}$ is the present day value of the Hubble parameter. Since in order to explain the cosmological ratio of the matter components $\varepsilon$ should have numerical values of the order of unity, it follows that the time changes of the dark matter and normal matter ratio are negligible small in the recent cosmological times. 


\section{Acknowledgments}

This work is supported by the RGC grant HKU 7027/06P of the Government of the Hong Kong SAR.

[1] P. Horava and E. Witten, Nucl. Phys. B460, 506 (1996).

[2] P. Horava and E. Witten, Nucl. Phys. B475, 94 (1996).

[3] J. Polchinski, Phys. Rev. Lett 75, 4724 (1995).

[4] L. Randall and R. Sundrum, Phys. Rev. Lett 83, 3370 (1999).

[5] L. Randall and R. Sundrum, Phys. Rev. Lett 83, 4690 (1999).

[6] M. Sasaki, T. Shiromizu and K. Maeda, Phys. Rev. D62, 024008 (2000); T. Shiromizu, K. Maeda and M. Sasaki, Phys. Rev. D62, 024012 (2000); K. Maeda, S. Mizuno and T. Torii, Phys. Rev. D68, 024033 (2003).

[7] P. Binétruy, C. Deffayet and D. Langlois, Nucl. Phys. B 565, 269 (2000); R. Maartens, Phys. Rev. D62, 084023 (2000); A. Campos and C. F. Sopuerta, Phys. Rev. D63, 104012 (2001); A. Campos and C. F. Sopuerta, Phys. Rev. D64, 104011 (2001); C.-M. Chen, T. Harko and M. K. Mak, Phys. Rev. D64, 044013 (2001); D. Langlois, Phys. Rev. Lett. 86, 2212 (2001); C.-M. Chen, T. Harko and M. K. Mak, Phys. Rev. D64, 124017 (2001); J. D. Barrow and R. Maartens, Phys. Lett. B532, 153 (2002); C.-M. Chen, T. Harko, W. F. Kao and M. K. Mak, Nucl. Phys. B64, 159 (2002); M. Szydlowski, M. P. Dabrowski and A. Krawiec, Phys. Rev. D66, 064003 (2002); T. Harko and M. K. Mak, Class. Quantum Grav. 20, 407 (2003); C.-M. Chen, T. Harko, W. F. Kao and M. K. Mak, JCAP 0311, 005 (2003); T. Harko and M. K. Mak, Class. Quantum Grav. 21, 1489 (2004); M. K. Mak and T. Harko, Phys. Rev. D 70, 024010 (2004); T. Harko and M. K. Mak, Phys. Rev. D69, 064020 (2004); A. N. Aliev and A. E. Gumrukcuoglu, Class. Quant. Grav. 21, 5081 (2004); M. Maziashvili, Phys. Lett. B627, 197 (2005); S. Mukohyama, Phys. Rev. D72, 061901 (2005); M. K. Mak and T. Harko, Phys. Rev. D71, 104022 (2005); A. N. Aliev and A. E. Gumrukcuoglu, Phys. Rev. D71, 104027, (2005); T. Harko and K. S. Cheng, Astrophys. J. 636, 8 (2006); L. A. Gergely, Phys. Rev. D74 024002, (2006); N. Pires, Zong-Hong Zhu, J. S. Alcaniz, Phys. Rev. D73, 123530 (2006); C. G. Böhmer and T. Harko, Class. Quantum Grav. 24, 3191 (2007); M. Heydari-Fard and H. 
R. Sepangi, Phys. Lett. B649, 1 (2007); T. Harko and K. S. Cheng, Phys. Rev. D76, 044013 (2007); A. Viznyuk and Y. Shtanov, Phys. Rev. D76, 064009 (2007); Z. Kovacs and L. A. Gergely, Phys. Rev. D77, 024003 (2008); T. Harko and V. S. Sabau, Phys. Rev. D77, 104009 (2008); M. V. Battisti, arXiv:0805.1178 (2008).

[8] D. N. Spergel et al., Astrophys. J. Supplement Series 170, 377 (2007).

[9] A. H. Guth, Phys. Rev. D71, 347 (1981).

[10] A. Linde, Phys. Repts. 333-334, 575 (2000); B. A. Bassett, S. Tsujikawa and D. Wands, Rev. Mod. Phys. 78, 537 (2006).

[11] A. D. Dolgov and A. D. Linde, Phys. Lett. B116, 329 (1982); L. F. Abbott, E. Farhi and M. B. Wise, Phys. Lett. B117, 29 (1982); A. Albrecht, P. J. Steinhardt, M. S. Turner and F. Wilczek, Phys. Rev. Lett. 48, 1437 (1982).

[12] M. Susperregi, Phys. Rev. D68, 123509 (2003).

[13] A. R. Liddle and L. A. Ureña-López, Phys. Rev. Lett. 97, 161301 (2006).

[14] V. H. Cárdenas, Phys. Rev. D75, 083512 (2007).

[15] E. W. Kolb, A. Notari and A. Riotto, Phys. Rev. D68, 123505 (2003).

[16] Y. Himemoto and T. Tanaka, Phys. Rev. D67, 084014 (2003).

[17] J. H. Brodie and D. A. Easson, JCAP 0312, 004 (2003).

[18] M. Sami, N. Dadhich and T. Shiromizu, Phys. Lett. B568, 118 (2003).

[19] Y. I. Takamizu and K. I. Maeda, Phys. Rev. D70, 123514 (2004).

[20] S. Hannestad, Phys. Rev. D70,043506 (2004).

[21] G. Panotopoulos, JCAP 0508, 005 (2005).

[22] E. Abou El Dahab and S. Khalil, JHEP 0609, 042 (2006).

[23] E. Papantonopoulos and V. Zamarias, JCAP 0611, 005 (2006).

[24] K. Umezu, K. Ichiki, T. Kajino, G. J. Mathews, R. Nakamura and M. Yahiro, Phys. Rev. D73, 063527 (2006).

[25] M. R. Setare, Phys. Lett. B642, 421 (2006); C. Bogdanos and K. Tamvakis, Phys. Lett. B646, 39 (2007).

[26] C. Bogdanos, S. Nesseris, L. Perivolaropoulos and K. Tamvakis, astro-ph 0705318.

[27] T. Harko and M. K. Mak, Astrophys. and Space Science, 253, 161 (1997).

[28] J. R. Ellis, J. E. Kim and D. V. Nanopoulos, Phys. Lett. B145, 181 (1984).

[29] M. Kawasaki and T. Moroi, Prog. Theor. Phys. 93, 879 (1995). 
[30] T. Moroi, hep-ph/9503210.

[31] P. S. Apostolopoulos and N. Tetradis, Phys. Rev. D71, 043506 (2005).

[32] E. Kiritsis, G. Kofinas, N. Tetradis, T. N. Tomaras and V. Zarikas, JHEP, 02, 035 (2003).

[33] N. Tetradis, Phys. Lett. B569, 1 (2003).

[34] P. S. Apostolopoulos and N. Tetradis, Class. Quant. Grav. 21, 4781 (2004).

[35] Y. S. Myung, J. Y. Kim, Class. Quant. Grav. 20, L169 (2003).

[36] N. Tetradis, Class. Quant. Grav. 21, 5221 (2004).

[37] P. S. Apostolopoulos and N. Tetradis, Phys. Rev. D71, 043506 (2005).

[38] S. Mukohyama, T. Shiromizu and K. I. Maeda, Phys. Rev. D D62, 024028 (2000).

[39] K. Ichiki, P. M. Garnavich, T. Kajino, G. L. Mathews and M. Yahiro, Phys. Rev. D68, 083518 (2003).

[40] A. R. Liddle and D. H. Lyth, Cosmological Inflation and Large-Scale Structure (Cambridge University Press, Cambridge, UK) (2000)

[41] E. W. Kolb and M. S. Turner, The Early Universe (Addison-Wesley, Menlo Park, CA) (1990).

[42] C. D. Hoyle et al., Phys. Rev. Lett. 86, 1418 (2001).

[43] N. Arkani-Hamed, S. Dimopolous, G. Dvali, N. Kaloper, Phys. Rev. Lett. 84, 586 (2000).

[44] D. J. H. Chung, L. Everett and H. Davoudiasl, Phys. Rev. D64, 065002 (2001).

[45] N. Okada and O. Seto, Phys. Rev. D71, 023517 (2005).

[46] A. Mazumdar, Nucl. Phys. B597, 561 (2000); A. Mazumdar, Phys. Rev. D64, 027304 (2001).

[47] R. Allahverdi, A. Mazumdar and A. Perez-Lorenzana, Phys. Lett. B516, 431 (2001). 